\title{
A Method for Detection of Obstacle Using Line Laser and Camera
}

\author{
Miki Suetsugu, Kanako Kinoshita, Shiyuan Yang, Siichi Serikawa \\ Department of Electrical and Electronic Engineering, Kyushu Institute of Technology, Kitakyushu, Japan \\ *Corresponding Author: p108060m@ mail.kyutech.jp
}

\begin{abstract}
Recently, the need for unmanned transport robots has increased. However, it has some problems. The transfer robot have to have a determined route. In addition, if there is a package on the traveling route, it has a possibility of collision. Thus, a technology for obstacle detection is important to avoid collision. As obstacle detection sensors recently, ultrasonic sensors, PSD sensors, and cameras are often used. However, there are several disadvantages. Ultrasonic sensors include errors due to sound noise, so they should be used in a quiet environment. The measurable distance of the PSD sensor is narrow. In addition, the sensor malfunctions due to irregular reflection from the floor. As for a camera, as it is necessary to sequentially update the background image, the calculation cost is high, and the detection accuracy is not high as compared with other sensors. To solve the problems, this study proposes an obstacle detection method using a line laser and a camera.

This method can detect obstacles over a wide range. It can calculate the width of obstacles with simple calculation. It has the following advantages in comparison with the conventional method.
\end{abstract}

(1) Simple image processing.

(2) No affection by fluctuations in lighting and noise.

(3) Simple calibration.

Therefore, it can greatly reduce preliminary work.

Keywords: Robot, Sensor, Image processing, Camera, Line Laser, Detection, Measurement.

\section{Introduction}

Recently, the use of online shopping is increasing in the world. In order to support this, it is important to accurately. Many of the transfer robots which works in the warehouse can move through the determined route. It is difficult to change the route. In addition, if there is a package on the route, it may happen collision. In the warehouse, there are many situations where humans and robots work together. Although humans sometimes move irregularly, robots cannot predict such a behavior. In order to avoid, it is necessary to detect obstacles in a wide range soon.

As obstacle detection sensors recently, ultrasonic sensors, PSD sensors, and cameras are often used. However, there are several disadvantages. Ultrasonic sensors include errors due to sound noise, so they should be used in a quiet environment. The measurable distance of the PSD sensor is narrow. In addition, the sensor malfunctions due to irregular reflection from the floor. As for a camera, as it is necessary to sequentially update the background image, the calculation cost is high, and the detection accuracy is not high as compared with other sensors.

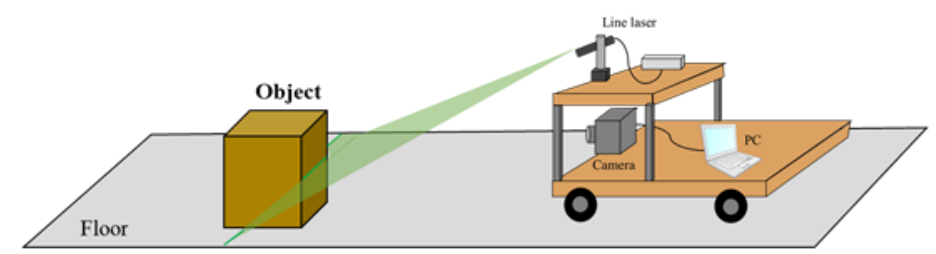

Figure 1: Outline of system configuration in this study.

To solve the problems, this study proposes an obstacle detection method using a line laser and a camera. This method can detect obstacles over a wide range. It can calculate the width of obstacles with simple calculation.

\section{How to detect obstacle detection by the use of line laser and camera}

The outline of the system configuration is shown in Figure 1. As understood from Figure 1, a green line laser is irradiated on the floor and the image is captured by a 
camera.

\subsection{Principle to detect obstacle}

\subsubsection{Extraction of laser line}

The laser line as shown in Figure 2 is extracted by preprocessing. In this study, an image captured by camera is converted from RGB color space to HSV color space. Next, the green areas are extracted. Since the color of laser is green, the area of laser line is included in the extracted areas. The area other than the line laser is easily erased by mask processing. Therefore, the area of laser is quickly and accurately extracted.

\subsubsection{Detection of Obstacle Detection by Line Laser}

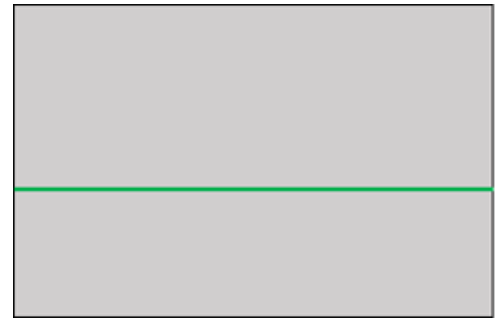

(a) Image when there is no obstacle

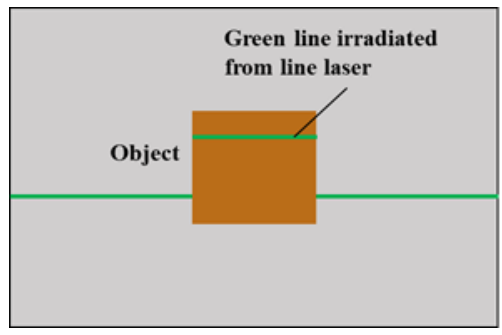

(b) Image when there is an obstacle

Figure2: Example of line laser after image processing

Figure 2(a) shows an image when there is no obstacle. When there is an obstacle, the line laser line is interrupted halfway as shown in Figure 2(b).

\subsection{How to calculate distance till obstacle}

How to calculate the height of the obstacle is mentioned in this sections. After that, the distance between the robot and the obstacle is obtained from the obtained height.

\subsubsection{Calculation of height of obstacle}

Here, let the variable of the obstacle height be $V_{r}[\mathrm{~cm}]$ in Figure 3. Let the distance between the obstacle and the robot be $\mathrm{D}[\mathrm{cm}]$, and let the distance between the destination irradiated to the floor and the robot be $\mathrm{X}[\mathrm{m}]$.

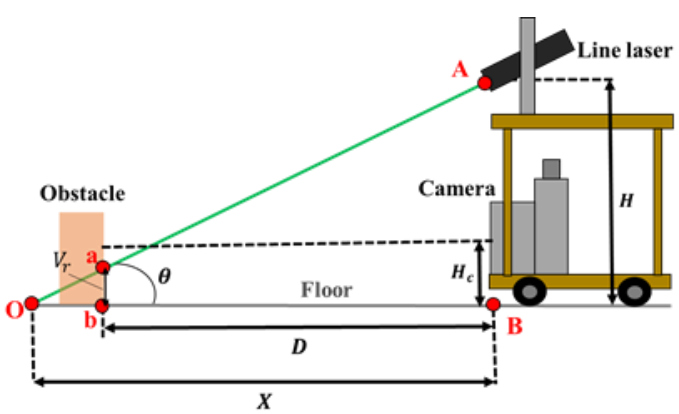

Figure3: Variable definition of obstacle height and distance

Let the height of the line laser be $\mathrm{H}$, let the height of the center of the camera lens be $H_{c}$. Letthe irradiation angle of the line laser be $\theta[\mathrm{deg}]$. Figure 4 shows an image extracted from positon shown in Figure 3.

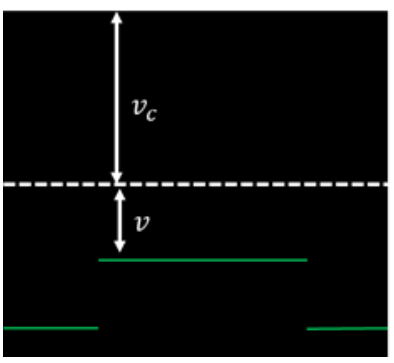

Figure4: Image of line laser extracted from camera.

Here, let the half size of the height be $v_{c}$ [pixel] and let the height from the center of the image to the line laser irradiated to the obstacle be $\mathrm{v}$ [pixel].

Figure 5 shows the relationship between an object, a lens, and an image sensor of a camera. Let the vertical size of the image pickup device of the camera be $\mathrm{y}[\mathrm{mm}]$ and the focal length be $\mathrm{f}[\mathrm{mm}]$.

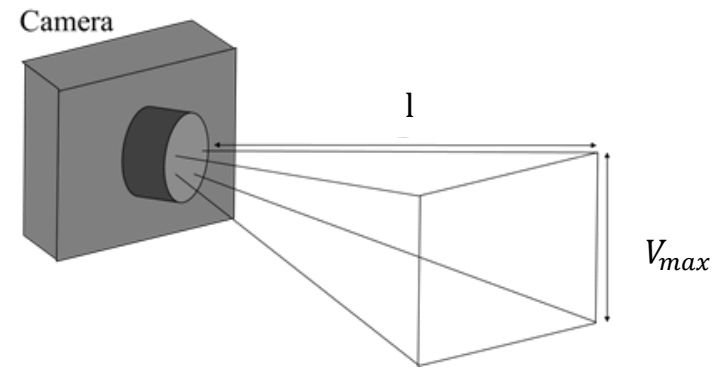

Figure5: Camera image sensor, object, lens relationship

In Figure 5, the vertical range $V_{\max }[\mathrm{m}]$ is expressed by 
equation (1).

$$
V_{\max }=\frac{l \times y}{f}[\mathrm{~m}]
$$

Since $V_{\max }$ is proportional to $v_{c}$, we can get the following relationship.

$$
\frac{V_{\max }}{V_{r}}=\frac{2 v_{c}}{v_{c}-v}
$$

The equation (2) is transformed as follows.

$$
V_{r}=\frac{V_{\max } \times\left(v_{c}-v\right)}{2 v_{c}} \times 100 \quad[\mathrm{~cm}]
$$

Thus, the height $V_{r}$ is obtained.

\subsubsection{Calculation of distance between obstacle and robot}

We can get the distance D between the obstacle and the robot by $V_{r}$. From the irradiation angle $\theta$ in Figure 3, D is obtained as follows.

$$
\mathrm{D}=\mathrm{X}-V_{r} \tan \theta
$$

Thus, the distance between obstacle and robot is gotten.

\section{Experiments}

\subsection{Experimental system}

The outline of the experimental system is explained by the use of Figure 5. The system consists of line laser, camera and personal computer which is connected to camera. The camera is placed at the lower position, and the line laser is set at the higher position. The light of line laser is irradiated on the floor. The image is captured with the camera and it is sent to the computer.

In this study, a line laser with a wavelength of 520 [mm] is adopted. The type is MLXL series made by KIKOH GIKEN. The camera of EOS M 10 made by Canon is used.

\subsection{Detection of height of obstacle}

In this experiment, the height of the obstacle is gotten by the Equation (2). The distance $\mathrm{L}$ between the destination of the line laser and the robot was changed by 1 $\mathrm{m}, 1.5 \mathrm{~m}$, and $2 \mathrm{~m}$. We measured the height by changing the width of the obstacle from $10 \mathrm{~cm}$ to $50 \mathrm{~cm}$. As understood from Figure 6, only the area of line laser is gotten from the image.

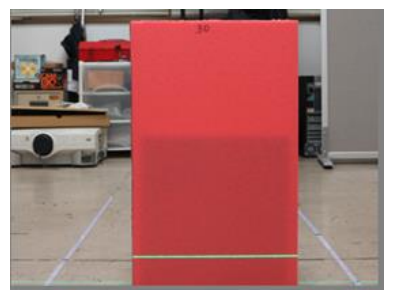

(a) Original image taken with camera

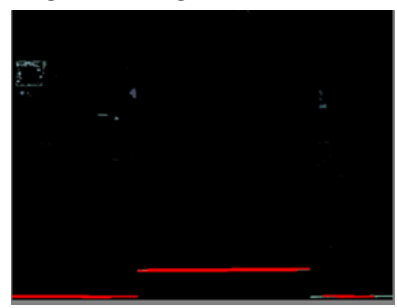

(b) Line extracted from the image (a)

\section{Figure6: Extraction of line laser from original image}

The error between the measured value and the theoretical value is shown in Figure 7, where symbol W means the width of the obstacle, and symbol D represents the distance between the robot and the obstacle.

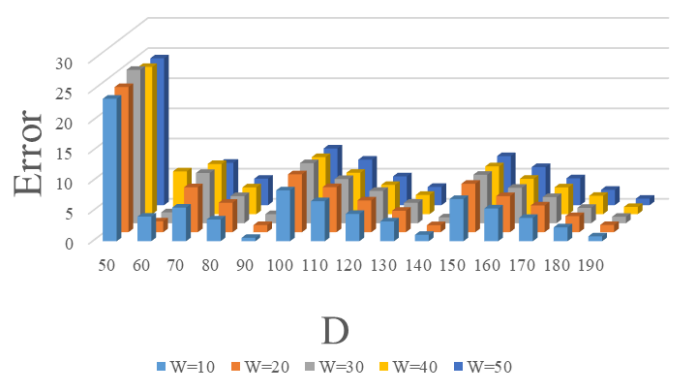

Figure 7: Measurement errors of $V_{r}$

This is the result when $D=50$ to $90 \mathrm{~cm}$ is set to $X=1 \mathrm{~m}$. In addition, the result when $\mathrm{D}=100$ to $140 \mathrm{~cm}$ is $\mathrm{X}=1.5 \mathrm{~m}$, and the case of $D=150$ to $190 \mathrm{~cm}$ is the result when $X=2$ $\mathrm{m}$. From figure 7 , we can understand that there is no difference in height measurement, even though the obstacle width $\mathrm{W}$ is changed. Thus, we can measure the height of the obstacle correctly. The error reached maximum when D $=50 \mathrm{~cm}$, and the error increases according as the distance between the obstacle and the robot decreases. The magnification of camera lens causes the difference of errors.

Table 1: Calculation error of height $V_{r}$

\begin{tabular}{|c|c|c|}
\hline$X[\mathrm{~m}]$ & Maximum error $[\mathrm{cm}]$ & Average error $[\mathrm{cm}]$ \\
\hline 1 & 25.4 & 8.13 \\
\hline 1.5 & 8.94 & 4.16 \\
\hline 2 & 8.13 & 4.34 \\
\hline
\end{tabular}


The details should be examined more. For $X=1 \mathrm{~m}$ the average of error was $8.13 \mathrm{~cm}$ and for $\mathrm{X}=2 \mathrm{~m}$ the average of error was $4.34 \mathrm{~cm}$. From Table 1, the average error is the smallest when $\mathrm{X}=1.5 \mathrm{~m}$.

\subsection{Detection of distance between obstacle and robot}

The distance $\mathrm{X}$ between the obstacle and the robot is calculated by the Equation (4). The distance D is gotten by changing the obstacle width $\mathrm{W}$ in the range from $10 \mathrm{~cm}$ to $50 \mathrm{~cm}$.

The error between the theoretical value and detected value is shown in Figure 8.

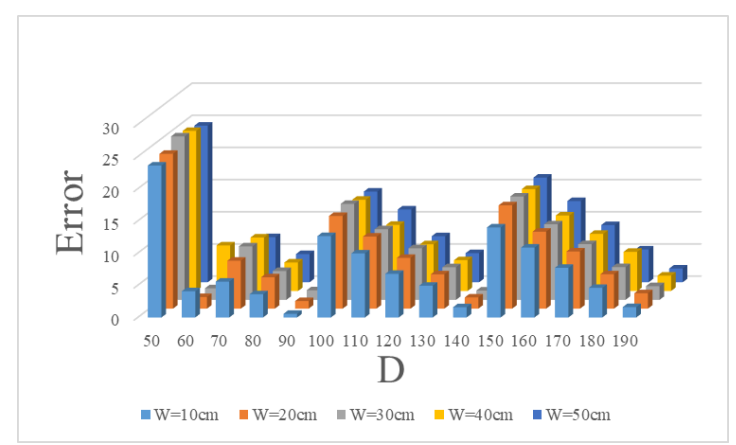

Figure 8: Measurement errors of D

From the figure, we can understand that there is no difference in error even if the width of the obstacle changes. The magnification of camera lens causes the difference of errors. It is necessary to correct the difference in lens magnification.

Table 2: Calculation error of distance D

\begin{tabular}{|c|c|c|}
\hline$X[\mathrm{~m}]$ & Maximum error $[\mathrm{cm}]$ & Average error $[\mathrm{cm}]$ \\
\hline 1 & 25.4 & 8.15 \\
\hline 1.5 & 14.9 & 7.74 \\
\hline 2 & 16.3 & 8.69 \\
\hline
\end{tabular}

From Table 2, the accuracy is good when $\mathrm{X}=1.5 \mathrm{~m}$. Correcting the perspective of the camera reduces the maximum error and further improves accuracy. If $\mathrm{X}=1.5$ $\mathrm{m}$ or more, the robot can detect obstacles and avoid them.

\section{Conclusions}

The need for unmanned transport robots is increasing. In order to use robots safely, obstacle detection technology is necessary to avoid collision with obstacles. In this study, we propose an obstacle detection method using line laser and camera. This method solves and improves the problem of the conventional method.
This method can detect obstacles over a wide range. It can calculate the width of obstacles with simple calculation. It has the following advantages in comparison with the conventional method. (1) Simple image processing. (2)No affection by fluctuations in lighting and noise. (3) Simple calibration.

As a future plans, I would like to improve the accuracy more and to enable stereoscopic detection of the depth of obstacles.

\section{References}

(1) Han Wang, Zhuo Wei, Sisong Wang, Chek Seng Ow, Kah Tong Ho and Benjamin Feng: "A vision-based obstacle detection system for Unmanned Surface Vehicle", IEEE, 5, pp.364-369, 2011

(2) Jeong-Gi Lee, D.Kim, Jin-Young Suk: "Development of a 3D Position Determination Device using PSD sensor", IEEE, pp.1733-1736, 2010

(3) Kosei Matsumoto, Toshio Moriya and Kazuo Yano: "Obstacle avoidance system for a user guided mobile robot”, United States Patent, pp.245-264, 2011

(4) Hidenori Ishihara, Toshio Fukuda, Kazuhiro Kosuge and F.Arai : "An approach to autonomous microrobot micro line trace robot with reflex algorithm", IEEE /ASME Transactions, Vol. 1, No. 1, pp.68-79, 1996

(5) Oe, Motoko, Tomokazu Sato, Naokazu Yokoya: "Estimating camera position and posture by using feature landmark database.”, Scandinavian Conference on Image Analysis. Springer Berlin Heidelberg, pp. 171-181, 2005

(6) Joydeep Biswas and Manuela Veloso: "Depth camera based indoor mobile robot localization and navigation", IEEE, pp.1967-1702, 2012

(7) Nils Gageik, Paul Benz and Sergio Montenegro: "Obstacle Detection and Collision Avoidance for a UAV With Complementary Low-Cost Sensors", IEEE, Volume3, pp.599-609, 2015

(8) Florin Oniga, Sergiu Nedevschi: "Processing Dense Stereo Data Using Elevation Maps: Road Surface, Traffic Isle, and Obstacle Detection", IEEE, 59, pp.1172-1182, 2009 